TRANSACTIONS OF THE

AMERICAN MATHEMATICAL SOCIETY

Volume 359, Number 4, April 2007, Pages 1593-1604

S 0002-9947(06)03935-3

Article electronically published on October 16, 2006

\title{
LATTICE-ORDERED ABELIAN GROUPS AND SCHAUDER BASES OF UNIMODULAR FANS
}

\author{
CORRADO MANARA, VINCENZO MARRA, AND DANIELE MUNDICI
}

\begin{abstract}
Baker-Beynon duality theory yields a concrete representation of any finitely generated projective Abelian lattice-ordered group $G$ in terms of piecewise linear homogeneous functions with integer coefficients, defined over the support $|\Sigma|$ of a fan $\Sigma$. A unimodular fan $\Delta$ over $|\Sigma|$ determines a Schauder basis of $G$ : its elements are the minimal positive free generators of the pointwise ordered group of $\Delta$-linear support functions. Conversely, a Schauder basis $\mathbf{H}$ of $G$ determines a unimodular fan over $|\Sigma|$ : its maximal cones are the domains of linearity of the elements of $\mathbf{H}$. The main purpose of this paper is to give various representation-free characterisations of Schauder bases. The latter, jointly with the De Concini-Procesi starring technique, will be used to give novel characterisations of finitely generated projective Abelian lattice ordered groups. For instance, $G$ is finitely generated projective iff it can be presented by a purely lattice-theoretical word.
\end{abstract}

\section{BACKGROUND: $\ell$-GROUPS AND FANS}

We assume familiarity with lattice-ordered Abelian groups (for short, $\ell$-groups [4, 7]) and fans. By a fan we shall always understand a finite rational polyhedral fan, as defined in [6, 15].

Throughout the paper, $\mathbb{N}=\{1,2, \ldots\}, \mathbb{Z}$ is the set of integers, and $\mathbb{R}$ is the set of reals. If $G$ is an $\ell$-group, we let $G^{+}=\{g \in G \mid g \geq 0\}$. By $\ell$-homomorphisms we mean homomorphisms of $\ell$-groups; the symbol ' $\cong$ ' denotes $\ell$-isomorphism. Kernels of $\ell$-homomorphisms are precisely $\ell$-ideals, always denoted by Gothic letters $\mathfrak{a}, \mathfrak{m}, \mathfrak{p}, \ldots$. An $\ell$-ideal is principal iff it is finitely generated (which for $\ell$-groups is equivalent to being singly generated). Maximal $\ell$-ideals are defined in the obvious manner. A finitely generated $\ell$-group $G$ is Archimedean iff it has no "infinitesimal elements": thus, whenever $0<x \leq y$ holds, there is $n \in \mathbb{N}$ such that $n x \not \leq y$; equivalently, the intersection of all maximal $\ell$-ideals in $G$ is $\{0\}$; see [7, 4.1] and 4. 10.2] for details. An $\ell$-ideal $\mathfrak{m}$ is maximal in $G$ iff $G / \mathfrak{m}$ is Archimedean totally ordered. As explained in [4, 13.2.6], Archimedean $\ell$-groups with a strong order unit (that is, an element $u \in G$ such that for every $x \in G$ there is $n \in \mathbb{N}$ with $n u \geq x$ ) are precisely those representable as $\ell$-groups of real-valued continuous functions over some compact Hausdorff space (operations being defined pointwise); finitely generated $\ell$-groups may always be endowed with a strong order unit.

Received by the editors March 30, 2004 and, in revised form, January 19, 2005.

2000 Mathematics Subject Classification. Primary 06F20, 52B20, 08B30; Secondary 06B25, $55 \mathrm{~N} 10$.

Key words and phrases. Lattice-ordered Abelian group, unimodular fan, projective $\ell$-group, De Concini-Procesi starring, singular homology group.

(C)2006 American Mathematical Society 
Suppose $G$ is a finitely generated $\ell$-group. Equipped with the spectral topology, the set $\operatorname{MaxSpec}(G)$ of maximal $\ell$-ideals of $G$ is a (nonempty) compact Hausdorff space. The closed sets in $\operatorname{MaxSpec}(G)$ are given by the zero sets $\mathfrak{Z}(S)$ of arbitrary subsets $S$ of $G$, where $\mathfrak{Z}(S)=\bigcap_{g \in S}\{\mathfrak{m} \in \operatorname{MaxSpec}(G) \mid g \in \mathfrak{m}\}$.

We let $|\Sigma| \subseteq \mathbb{R}^{n}$ denote the (closed) support of the fan $\Sigma 11$ A continuous function $f:|\Sigma| \rightarrow \mathbb{R}$ is said to be an (integral) $\ell$-function over $|\Sigma|$ iff it is (always finitely) piecewise linear homogeneous, each piece having the form $c_{1} x_{1}+\cdots+c_{n} x_{n}$, for suitable integers $c_{1}, \ldots, c_{n}$.

By $\mathcal{A}_{n}$ we denote the $\ell$-group of all $\ell$-functions over $\mathbb{R}^{n}$, with pointwise operations. As is well known, $\mathcal{A}_{n}$ is free in the equational class of $\ell$-groups. The (coordinate) projection functions $\pi_{i}: \mathbb{R}^{n} \rightarrow \mathbb{R}$ are free generators of $\mathcal{A}_{n}$. For $\Sigma$ a fan in $\mathbb{R}^{n}$, we let

$$
\mathcal{A}_{n} \uparrow|\Sigma|
$$

denote the $\ell$-group of all $\ell$-functions over $|\Sigma|$. This notation is in agreement with the fact that $\ell$-functions over $|\Sigma|$ are the same as restrictions to $|\Sigma|$ of $\ell$-functions over $\mathbb{R}^{n}$.

Elements of fans are called cones; thus, a cone is always a rational polyhedral cone. A fan $\Theta$ is simplicial iff every one of its cones is simplicial (i.e., taking an affine section in general position yields a simplex). A simplicial cone $\sigma \in \Theta$ is unimodular iff its 1-dimensional faces are spanned (over the positive reals) by primitive vector 2 that are columns of some unimodular matrix (an integral square matrix with determinant \pm 1 ). A fan is unimodular iff all of its cones are unimodular 3

Given two fans $\Sigma$ and $\Delta$, if $|\Delta|=|\Sigma|$ and all the cones of $\Sigma$ are unions of cones of $\Delta$, then we say that $\Delta$ refines $\Sigma$.

For $\Delta$ a unimodular fan in $\mathbb{R}^{n}$, let $\sigma \in \Delta$ be a 1-dimensional cone. Following 12, 13. (also see [17), the Schauder hat of $\Delta$ at $\sigma$ is the unique $\ell$-function $h_{\sigma}$ that is linear over each cone of $\Delta$, has value 1 at the primitive vector along $\sigma$, and value 0 at every point of every other 1-dimensional cone of $\Delta$. The Schauder basis over $\Delta$, denoted Hats $\Delta$, is the collection of all Schauder hats of $\Delta$. Integrality of the linear pieces is guaranteed by unimodularity of the fan.

Every Schauder hat of $\Delta$ is a particular case of a $\Delta$-linear support function [15, page 66]. Specifically, the Schauder basis over $\Delta$ is a free generating set in the free group $\mathcal{F}$ of $\Delta$-linear support functions. If, in addition, $\mathcal{F}$ is equipped with the pointwise order, then the Schauder hats over $\Delta$ are just the minimal positive free generators; see [13.

\section{Schauder bases and De Concini-Procesi Refinements}

A nontrivial exercise shows that there are $\ell$-automorphisms of, say, $\mathcal{A}_{3}$, carrying a Schauder basis to a set of $\ell$-functions which is not a Schauder basis. Thus, the property of being a Schauder basis is not invariant under $\ell$-automorphisms.

A useful representation-independent generalisation is the following.

Definition 2.1. Let $G$ be an $\ell$-group and $B=\left\{b_{1}, \ldots, b_{t}\right\} \subseteq G$, where $t \in \mathbb{N}$. We say that $B$ is an abstract Schauder basis of $G$ iff for some $n \in \mathbb{N}$ there exist a unimodular fan $\Delta$ in $\mathbb{R}^{n}$, and an $\ell$-isomorphism $\phi: \mathcal{A}_{n} \uparrow|\Delta| \cong_{\ell} G$, such that,

\footnotetext{
${ }^{1}$ All fans are denoted by capital Greek letters.

${ }^{2} \mathrm{An}$ integral vector is primitive iff the greatest common divisor of its coordinates is 1 .

${ }^{3}$ Unimodular fans are also known as nonsingular fans, as they correspond precisely to smooth toric varieties under the fan-toric dictionary. In [6], they are called regular fans.
} 
letting $\operatorname{Hats}_{\Delta}=\left\{h_{1}, \ldots, h_{t}\right\}$ be the Schauder basis over $\Delta$, we have $\phi\left(h_{i}\right)=b_{i}$ for every $i \in\{1, \ldots, t\}$ 迎

When we wish to emphasise that $B$ is a Schauder basis, we sometimes write that it is a concrete Schauder basis. Trivially, any concrete Schauder basis is an abstract Schauder basis.

Proposition 2.2. If the $\ell$-group $G$ has an abstract Schauder basis B, then

(1) $B \subseteq G^{+} \backslash\{0\}$,

(2) $B$ is linearly independent in the $\mathbb{Z}$-module $G$, and

(3) $G$ is Archimedean.

Proof. In view of the existence of the above $\ell$-isomorphism $\phi: \mathcal{A}_{n} \uparrow|\Delta| \cong_{\ell} G$, it is enough to prove the proposition for $\mathcal{A}_{n} \uparrow|\Delta|$ equipped with the concrete Schauder basis Hats $\Delta=\left\{h_{1}, \ldots, h_{t}\right\}$ corresponding to $B$ via $\phi$. Then (1) and (2) are immediate. Property (3) follows upon observing that $\mathcal{A}_{n} \uparrow|\Delta|$ is an $\ell$-group of real-valued functions.

In Proposition 2.5 below we shall prove that $B$ generates $G$. The proof requires some background material on stellar subdivisions [6, 15. Barycentric stellar subdivisions (starrings for short) can be used to subdivide a unimodular fan, preserving unimodularity 5 If $\Delta$ is obtained from $\Sigma$ via a finite number (possibly zero) of starrings, we write $\Delta \preceq \Sigma$; and if all such subdivisions are along 2-dimensional cones only (binary starrings for short), we write $\Delta \preceq_{2} \Sigma$.

Lemma 2.3 (The De Concini-Procesi Lemma). Let $\Sigma_{1}$ and $\Sigma_{2}$ be unimodular fans with the same support. There exists a unimodular fan $\Delta$ such that $\Delta \preceq_{2} \Sigma_{1}$ and $\Delta$ refines $\Sigma_{2}$.

Proof. See [5]; for a short elementary proof, see [16] or [13].

For our purposes, it is crucial to realise that starrings of unimodular fans are definable in the language of $\ell$-groups. Although this is really a remark, we record it for easier reference.

Lemma 2.4. Let $\Sigma$ be a unimodular fan and $\mathbf{H}=\mathrm{Hats}_{\Sigma}$ its associated Schauder basis. Let $h_{1}, h_{2} \in \mathbf{H}$ be hats at the 1 -cones $\sigma_{1}$ and $\sigma_{2}$, respectively. Suppose $\sigma_{1}$ and $\sigma_{2}$ span a 2-cone $\tau$ of $\Sigma$ (equivalently, $h_{1} \wedge h_{2} \neq 0$ ). Let $\Delta \preceq_{2} \Sigma$ be the unimodular fan obtained from $\Sigma$ by starring along $\tau$. Then $\mathbf{K}=$ Hats $_{\Delta}$ is the Schauder basis obtained from $\mathbf{H}$ by removing $h_{1}$ and $h_{2}$, and adjoining the three hats

$$
h_{1}-\left(h_{1} \wedge h_{2}\right), h_{2}-\left(h_{1} \wedge h_{2}\right), h_{1} \wedge h_{2} .
$$

Conversely, if $\mathbf{K}$ is obtained from $\mathbf{H}$ by such substitutions, then there is a unique unimodular fan $\Delta$, obtained from $\Sigma$ by starring along $\tau$, such that $\mathbf{K}=$ Hats $_{\Delta}$.

Proof. A straightforward computation.

Proposition 2.5. If an $\ell$-group $G$ has an abstract Schauder basis $B$, then $B$ generates $G$.

\footnotetext{
${ }^{4}$ In Proposition 2.5 below we shall prove that if $G$ has a Schauder basis, then $G$ is finitely generated.

${ }^{5}$ Starrings correspond to (equivariant) blow-ups of toric varieties via the fan-toric dictionary [15, 6].
} 
Proof. Using the $\ell$-isomorphism $\phi: \mathcal{A}_{n} \uparrow|\Delta| \cong_{\ell} G$, without loss of generality we can assume $G=\mathcal{A}_{n} \uparrow|\Delta|$ equipped with the concrete Schauder basis $B=$ Hats $_{\Delta}=$ $\left\{h_{1}, \ldots, h_{t}\right\}$. By [6, Theorem 8.5], for every $\ell$-function $f$ over $|\Delta|$, there exists a unimodular refinement $\Delta_{f}$ of $\Delta$ such that $f$ is linear over every cone of $\Delta_{f}$. By Lemma 2.3, an appropriate sequence of binary starrings transforms $\Delta$ into a refinement $\Delta^{*}$ of $\Delta_{f}$. By Lemma 2.4, all hats of $\Delta^{*}$ are in the $\ell$-group $H$ of realvalued functions over $|\Delta|$ generated by $B$. Since, by the construction of $\Delta^{*}, f$ must take an integer value at each primitive generating vector of $\Delta^{*}$, then $f$ is a linear combination of the hats of $\Delta^{*}$ with integer coefficients. This shows that $H=\mathcal{A}_{n} \uparrow|\Delta|$.

The concept of concrete Schauder bases only makes sense for $\ell$-groups of the form $G=\mathcal{A}_{n} \uparrow|\Delta|$, where $|\Delta|$ is a unimodular fan. The main question we shall address is whether one can characterise abstract Schauder bases without reference to their geometric realisations.

\section{Topological characterisation of abstract Schauder bases}

Let $G$ be an $\ell$-group, and $D$ be a finite subset of $G$. Then by an abstract $k$-simplex of $D$ we mean a set $Y \subseteq D$ of cardinality $k+1$ such that $\bigwedge Y \neq 0$.

Theorem 3.1. Let $G$ be an $\ell$-group, and let $B=\left\{b_{1}, \ldots, b_{t}\right\} \subseteq G^{+} \backslash\{0\}$ be a finite subset. Then the following conditions are equivalent:

(1) $B$ is an abstract Schauder basis of $G$.

(2) $G$ is Archimedean, $B$ generates $G$, and for every abstract $k$-simplex $Y$ of $B$, the zero set $\mathfrak{Z}(B \backslash Y)$ is homeomorphic to the closed $k$-dimensional ball.

Proof.6 $(2 \rightarrow 1) \quad$ Let the $\ell$-homomorphism $\eta: \mathcal{A}_{t} \rightarrow G$ be the unique extension of the map $\pi_{i} \mapsto b_{i} \quad(i=1, \ldots, t)$. Then $\eta$ is surjective, because $B$ generates $G$. Since $G$ is Archimedean, $G$ is $\ell$-isomorphic to the $\ell$-group $\mathcal{A}_{t} \uparrow W$ of restrictions of the $\ell$-functions of $\mathcal{A}_{t}$ to a suitable homogeneous 7 closed subset $W$ of $\mathbb{R}^{t}$. We shall show that $W$ is the support of a unimodular fan $\Theta$, and $B$ is the $\eta$-image of Hats $\Theta$.

The assumption that each $b_{i}$ is positive implies that $W$ is contained in the positive orthant $\mathcal{O}$ of $\mathbb{R}^{t}$. Let $L$ be the affine hyperplane determined by the unit basic vectors $\mathbf{e}_{i}$,

$$
L=\left\{\left(\xi_{1}, \ldots, \xi_{t}\right) \in \mathbb{R}^{t} \mid \sum \xi_{i}=1\right\} .
$$

Let $W^{\prime}=W \cap L$. Via the canonical $\ell$-isomorphism of $\mathcal{A}_{t} \uparrow W$ onto $\mathcal{A}_{t}\left\lceil W^{\prime}\right.$, we shall identify $G$ with the $\ell$-group of restrictions to $W^{\prime}$ of the functions of $\mathcal{A}_{t}$; in symbols,

$$
G=\mathcal{A}_{t} \uparrow W^{\prime} .
$$

Under this identification, $\eta$ is just the restriction map, and every element $b_{i} \in B$ coincides with the restriction to $W^{\prime}$ of the $i$ th coordinate function $\pi_{i}$ of $\mathcal{A}_{t}$. Since the rays of $W$ are in one-one correspondence with the maximal $\ell$-ideals of $G$, there also is a natural one-one correspondence

$$
\mathfrak{m} \in \operatorname{Max} \operatorname{Spec}(G) \mapsto \mathbf{z}_{\mathfrak{m}} \in W^{\prime}
$$

\footnotetext{
${ }^{6}$ The techniques for the proof of Theorem 3.1 were developed, in a different but related context, in [14. For partitions of unity, a similar result is stated in 9 .

${ }^{7}$ Meaning that whenever $W$ contains a point $\mathbf{z}$, then $W$ also contains the ray through 0 and $\mathbf{z}$.
} 
between maximal $\ell$-ideals $\mathfrak{m}$ and points in $W^{\prime} 8$ This correspondence is also a homeomorphism of $\operatorname{MaxSpec}(G)$ (equipped with the spectral topology; see 4, Chapter 13]) onto $W^{\prime}$ (with the natural topology of $\mathbb{R}^{t}$ ). Accordingly, we shall identify MaxSpec $(G)$ and $W^{\prime}$ as topological spaces. The quotient map $f \in G \mapsto$ $f / \mathfrak{m} \in G / \mathfrak{m}$ coincides with the evaluation map of every $f \in G$ at the point $\mathbf{z}_{\mathfrak{m}}=$ $\left(\xi_{1}, \ldots, \xi_{t}\right)$ corresponding to the maximal $\ell$-ideal $\mathfrak{m}$; in symbols,

$$
f / \mathfrak{m}=f\left(\mathbf{z}_{\mathfrak{m}}\right), \text { for all } \mathfrak{m} \in \operatorname{MaxSpec}(G) .
$$

Claim 1. Let $Y=\left\{b_{i_{1}}, \ldots, b_{i_{k}}\right\}$ be an abstract simplex of $B$. Then the zero set $\mathfrak{Z}(B \backslash Y)$ coincides with the set $\left\{\mathbf{z} \in W^{\prime} \mid b_{i_{1}}(\mathbf{z})+\cdots+b_{i_{k}}(\mathbf{z})=1\right\}$.

As a matter of fact, by (3), $\mathfrak{Z}(B \backslash Y)$ is the set of points of $W^{\prime}$ where all functions of $B \backslash Y$ vanish. On the other hand, the sum of all functions $b_{j}$ of $B$ (equivalently, the sum of the corresponding $\pi_{j}$ ) constantly equals 1 over $W^{\prime}$.

Claim 2. For each $i=1, \ldots, t$, the singleton $\left\{b_{i}\right\}$ forms an abstract 0 -simplex of $B$, and the zero set $\mathfrak{Z}\left(B \backslash\left\{b_{i}\right\}\right)$ is the (singleton) standard basis vector $\mathbf{e}_{i}$. Thus $\mathbf{e}_{i} \in W^{\prime}$.

By assumption $\left\{b_{i}\right\}$ is nonzero. By definition it forms an abstract 0 -simplex of $B$. By our topological assumption, $\mathfrak{Z}\left(B \backslash\left\{b_{i}\right\}\right)$ is a singleton in the compact Hausdorff space $\operatorname{MaxSpec}(G)=W^{\prime}$. The only possible point $\mathbf{z}$ in $\mathcal{O} \cap L$ where $b_{i}(\mathbf{z})=1$ is the basis vector $\mathbf{e}_{i}$. Thus, by Claim $1, \mathbf{e}_{i} \in W^{\prime}$.

Claim 3. For every abstract $(k-1)$-simplex $Y=\left\{b_{i_{1}}, \ldots, b_{i_{k}}\right\}$ of $B$, the zero set $\mathfrak{Z}(B \backslash Y)$ is the convex hull $\left[\mathbf{e}_{i_{1}}, \ldots, \mathbf{e}_{i_{k}}\right]$ of the basis vectors $\mathbf{e}_{i_{1}}, \ldots, \mathbf{e}_{i_{k}}$. Thus, in particular, $\left[\mathbf{e}_{i_{1}}, \ldots, \mathbf{e}_{i_{k}}\right] \subseteq W^{\prime}$.

The proof is by induction on $k$.

Basis. Suppose $\left\{b_{i}, b_{j}\right\}$ forms an abstract 1-simplex $Y$ of $B$. By Claim 1, $\mathfrak{Z}(B \backslash Y)$ coincides with the set $V=\left\{\mathbf{z} \in W^{\prime} \mid b_{i}(\mathbf{z})+b_{j}(\mathbf{z})=1\right\}$. By definition of $W^{\prime}$, together with (3), $V$ is a subset of the closed segment $\left[\mathbf{e}_{i}, \mathbf{e}_{j}\right]$. By Claim 2, both $\mathbf{e}_{i}$ and $\mathbf{e}_{j}$ belong to $V$, because both $b_{i}$ and $b_{j}$ form an abstract 0 -simplex of $B$. If $V$ were a proper subset of $\left[\mathbf{e}_{i}, \mathbf{e}_{j}\right]$, then it would not be connected, against our topological assumption. Thus, $\mathfrak{Z}(B \backslash Y)=\left[\mathbf{e}_{i}, \mathbf{e}_{j}\right]$.

Induction step. Let $D=\left\{b_{i_{1}}, \ldots, b_{i_{k+1}}\right\}$ be an abstract $k$-simplex of $B$. A fortiori, every subset $D^{\prime}=\left\{b_{j_{1}}, \ldots, b_{j_{k}}\right\}$ of $D$ is an abstract $(k-1)$-simplex of $B$. By induction hypothesis, the zero set $\mathfrak{Z}\left(B \backslash D^{\prime}\right)$ coincides with the (topological) $(k-1)$-simplex $\left[\mathbf{e}_{j_{1}}, \ldots, \mathbf{e}_{j_{k}}\right]$. Thus, by (3), $\mathfrak{Z}(B \backslash D)$ is a certain subset $V \subseteq\left[\mathbf{e}_{i_{1}}, \ldots, \mathbf{e}_{i_{k+1}}\right]$, containing the union of $S^{k-1}$ all $(k-1)$-dimensional faces of $\left[\mathbf{e}_{i_{1}}, \ldots, \mathbf{e}_{i_{k+1}}\right]$. Suppose $V$ were a proper subset of $\left[\mathbf{e}_{i_{1}}, \ldots, \mathbf{e}_{i_{k+1}}\right]$ (absurdum hypothesis). Write

$$
V=\left[\mathbf{e}_{i_{1}}, \ldots, \mathbf{e}_{i_{k+1}}\right] \backslash U
$$

for a suitable nonempty subset $U$ of the relative interior of $\left[\mathbf{e}_{i_{1}}, \ldots, \mathbf{e}_{i_{k+1}}\right]$. As in [13], the intuitively obvious fact that $V$ cannot be homeomorphic to the $k$-ball is confirmed by the verification that the singular homology groups [1] of $V$ and of $\left[\mathbf{e}_{i_{1}}, \ldots, \mathbf{e}_{i_{k+1}}\right]$ are not isomorphic.

\footnotetext{
${ }^{8}$ One passes from $\mathfrak{m}$ to $\mathbf{z}_{\mathfrak{m}}$ by taking the intersection of all zero sets of functions in $\mathfrak{m}$. Conversely, one passes from any point $\mathbf{z} \in W^{\prime}$ to its corresponding maximal $\ell$-ideal $\mathfrak{m}$ by taking all functions of $G$ that vanish at $\mathbf{z}$.
} 
On the one hand, $H_{k-1}\left(\left[\mathbf{e}_{i_{1}}, \ldots, \mathbf{e}_{i_{k+1}}\right]\right)$ is zero, because $\left[\mathbf{e}_{i_{1}}, \ldots, \mathbf{e}_{i_{k+1}}\right]$ is contractible. On the other hand, letting $\mathbf{u}$ be any point in set $U$, the inclusion maps

$$
S^{k-1} \rightarrow V \rightarrow \mathbb{R}^{n} \backslash\{\mathbf{u}\}
$$

induce homomorphisms of homology groups

$$
\mathbb{Z}=H_{k-1}\left(S^{k-1}\right) \rightarrow H_{k-1}(V) \rightarrow H_{k-1}\left(\mathbb{R}^{n} \backslash\{\mathbf{u}\}\right)=\mathbb{Z} .
$$

Moreover, the composition of these two homomorphisms is the homomorphism induced by the inclusion

$$
S^{k-1} \rightarrow \mathbb{R}^{n} \backslash\{\mathbf{u}\}
$$

Because the latter map is a homotopy equivalence, it induces an isomorphism on homology groups. We have shown that $H_{k-1}(V)$ contains the group of integers as a summand. This contradicts our topological assumption, and also settles our claim.

To conclude the proof of $(2 \rightarrow 1)$, for each abstract $(k-1)$-simplex $Y$ of $B$ let $\sigma_{Y}$ be an abbreviation of the zero set $\mathfrak{Z}(B \backslash Y)$. By Claim $3, \sigma_{Y}$ is a $(k-1)$-dimensional simplex. For any two abstract simplices $Y^{\prime}, Y^{\prime \prime}$ of $B$, their corresponding simplices $\sigma_{Y^{\prime}}, \sigma_{Y^{\prime \prime}}$ intersect in a common face, namely the simplex $\sigma_{Y^{\prime} \cap Y^{\prime \prime}}$. Here it is convenient to write $\sigma_{\emptyset}=\emptyset$.

The abstract simplices of $B$ then determine a (concrete, topological) simplicial complex $\mathcal{S}$ in $\mathbb{R}^{t}$, and $W^{\prime}$ is the support of $\mathcal{S} 9$ The $t$ vertices of $\mathcal{S}$ are the standard basis vectors of $\mathbb{R}^{t}$. Further, for each $k=1, \ldots, t$, letting $\mathcal{S}^{(k)}$ denote the set of $k$-dimensional simplices of $\mathcal{S}$, we have the correspondence

$$
\mathcal{S}^{(k)} \longleftrightarrow \text { abstract } k \text {-simplices of } B \text {. }
$$

Replacing each point $\mathbf{z}$ in $W^{\prime}$ by the ray passing through 0 and $\mathbf{z}$, we obtain from $\mathcal{S}$ a unimodular fan $\Theta$ whose support coincides with $W$. The primitive generating vectors of $\Theta$ are the standard basis vectors $\mathbf{e}_{i}$. The Schauder hats of $\Theta$ are precisely the restrictions $\pi_{i} \uparrow W$, and hence they correspond to the elements of $B$, via our standing $\ell$-isomorphism $b_{i} \cong_{\ell} \pi_{i}\lceil W$.

This completes the proof of $(2 \rightarrow 1)$.

$(1 \rightarrow 2)$ For some $n \in \mathbb{N}$ and unimodular fan $\Delta$ there is an $\ell$-isomorphism

$$
\phi: \mathcal{A}_{n} \uparrow|\Delta| \cong_{\ell} G
$$

that geometrically realises $B$ by the Schauder basis over $\Delta$. By Propositions 2.2 and 2.5. $G$ is Archimedean and $B$ generates $G$. Without loss of generality we may assume $|\Delta|$ to lie entirely in the positive orthant of $\mathbb{R}^{n} 10$ Let $L$ be the hyperplane

$$
\left\{\left(\xi_{1}, \ldots, \xi_{n}\right) \in \mathbb{R}^{n} \mid \xi_{1}+\cdots+\xi_{n}=1\right\} .
$$

Let $L_{\Delta}=|\Delta| \cap L$ equipped with the natural topology. Then $\operatorname{MaxSpec}\left(\mathcal{A}_{n} \uparrow|\Delta|\right)$ is canonically homeomorphic to $L_{\Delta}$; let us identify these topological spaces. Since $\Delta$ is a complex of simplicial cones, its affine section $L_{\Delta}$ is a simplicial complex. For every abstract simplex $Y=\left\{f_{1}, \ldots, f_{k}\right\}$ of $B$, let $\mathbf{v}_{i} \in \mathbb{R}^{n}$ be the point of $L_{\Delta}$ where the Schauder hat $f_{i}$ attains its maximum value. Then direct inspection shows that the zero set $T=\mathfrak{Z}(B \backslash Y)$ is the convex hull in $\mathbb{R}^{n}$ of the points $\mathbf{v}_{1}, \ldots, \mathbf{v}_{k}$. Therefore, $T$ is homeomorphic to the $(k-1)$-dimensional ball.

The proof is complete.

\footnotetext{
${ }^{9}$ That is, $W^{\prime}$ is the set-theoretical union of all simplices of $\mathcal{S}$.

${ }^{10}$ Indeed, we can further assume that the primitive generating vectors of the rays of $\Delta$ coincide with the standard basis vectors $\mathbf{e}_{1}, \ldots, \mathbf{e}_{n}$ of $\mathbb{R}^{n}$.
} 


\section{Characterising Finitely Generated PROJECtive $\ell$-Groups}

As we have seen, abstract Schauder bases are in one-one correspondence with unimodular fans. In this section they are used to characterise projective $\ell$-groups.

By definition, an $\ell$-group $G$ is projective if, whenever $\varphi: H \rightarrow K$ is a surjective $\ell$ homomorphism and $\alpha: G \rightarrow K$ is an $\ell$-homomorphism, there is an $\ell$-homomorphism $\theta: G \rightarrow H$ such that $\varphi \circ \theta=\alpha$.

It follows that an $\ell$-group $G$ is finitely generated projective iff it is a retract of some $\mathcal{A}_{n}$. In other words, there are maps $\iota: G \rightarrow \mathcal{A}_{n}$ and $\sigma: \mathcal{A}_{n} \rightarrow G$ such that $\sigma \circ \iota$ is the identity over $G$.

The following well-known characterisation is due to Baker and Beynon [1, 2, 3.

Theorem 4.1 ([7, Corollary 5.2.2], 3, Theorem 3.1]). For any $\ell$-group $G$ the following conditions are equivalent:

(1) $G$ is finitely generated projective;

(2) for some $n \in \mathbb{N}, G$ is $\ell$-isomorphic to an $\ell$-group of $\ell$-functions over the support of some fan $\Sigma$ in $\mathbb{R}^{n}$, in symbols, $G \cong \mathcal{A}_{n} \uparrow|\Sigma|$; and

(3) for some $n \in \mathbb{N}, G$ is $\ell$-isomorphic to the quotient $\mathcal{A}_{n} / \mathfrak{p}$ for some principal $\ell$-ideal $\mathfrak{p}$ of $\mathcal{A}_{n}$.

\section{Hence:}

Proposition 4.2. For any $\ell$-group $G$ the following are equivalent:

(1) $G$ is finitely generated projective.

(2) G has an abstract Schauder basis.

Proof. $(1 \rightarrow 2)$ By Theorem 4.1 we can write $\psi: G \cong_{\ell} \mathcal{A}_{n} \uparrow|\Sigma|$ for some fan $\Sigma$ in $\mathbb{R}^{n}$. Now, every fan can be unimodularised (see, e.g., [6. Theorem 8.5]), hence its support is also the support of a unimodular fan. Accordingly, we shall assume that $\Sigma$ is unimodular. Let Hats $\Sigma$ be the concrete Schauder basis over $\Sigma$. The $\ell-$ isomorphism $\psi^{-1}$ induces a one-one correspondence between Hats $\Sigma$ and an abstract Schauder basis of $G$.

$(2 \rightarrow 1)$ If $G$ has an abstract Schauder basis, then $G \cong_{\ell} \mathcal{A}_{n} \uparrow|\Delta|$ for some unimodular fan $\Delta$. By Theorem 4.1, $\mathcal{A}_{n} \uparrow|\Delta|$ is finitely generated projective, hence so is $G$.

Now recalling Theorem 3.1 we immediately obtain from the proposition above:

Corollary 4.3. For any $\ell$-group $G$ the following are equivalent:

(1) $G$ is finitely generated projective.

(2) $G$ is Archimedean and is generated by a finite set $B \subseteq G^{+} \backslash\{0\}$ such that for every abstract $k$-simplex $Y$ of $B$, the zero set $\mathfrak{Z}(B \backslash Y)$ is homeomorphic to the $k$-ball.

The proof of Theorem 3.1, in conjunction with Proposition 4.2, actually yields the following interesting variant of $(1 \leftrightarrow 2)$ in Theorem 4.1.

Corollary 4.4. For any $\ell$-group $G$ the following are equivalent:

(1) $G$ is finitely generated projective.

(2) For some positive integer $n, G$ is -isomorphic to $\mathcal{A}_{n} \uparrow|\Theta|$, where $\Theta$ is a (necessarily unimodular) fan in $\mathbb{R}^{n}$ whose 1 -dimensional cones are generated by the standard basis vectors $\mathbf{e}_{1}, \ldots, \mathbf{e}_{n}$. 


\section{Refinements of ABStract Schauder Bases}

As shown in [6, 15], refinements of unimodular fans are fundamental to the fantheoretic treatment of birational toric geometry. In this section we derive abstract versions of two basic refinement procedures for unimodular fans.

Let $\Sigma$ and $\Delta$ be unimodular fans in $\mathbb{R}^{n}$ with the same support. Let [[Hats $\left.\left.{ }_{\Sigma}\right]\right]$ and

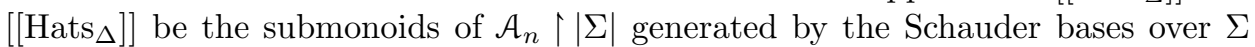
and $\Delta$, respectively. Direct inspection shows that $\Sigma$ refines $\Delta$, henceforth written $\Sigma \leq \Delta$, iff $\left[\left[\right.\right.$ Hats $\left.\left._{\Delta}\right]\right] \subseteq\left[\left[\right.\right.$ Hats $\left.\left._{\Sigma}\right]\right]$ iff Hats $_{\Delta} \subseteq\left[\left[\right.\right.$ Hats $\left.\left._{\Sigma}\right]\right]$. Thus the following proposition shows that, in a natural sense, any two abstract Schauder bases of the same $\ell$-group admit a joint refinement.

Proposition 5.1 (Joint refinability). Let $B_{1}, B_{2}$ be abstract Schauder bases of an $\ell$-group $G$. There exists an abstract Schauder basis $C$ of $G$ such that $C \leq B_{1}, B_{2}$, where $C \leq B_{i}$ stands for $B_{i} \subseteq[[C]]$, and $[[C]]$ is the submonoid of $G$ generated by C.

Proof. Let $\phi: G \cong_{\ell} \mathcal{A}_{n} \uparrow|\Sigma|$ be an $\ell$-isomorphism carrying $B_{1}$ to Hats $\Sigma$, for some unimodular fan $\Sigma$ in $\mathbb{R}^{n}$. Then $\phi$ carries $B_{2}$ to a collection of $\ell$-functions $S=\phi\left(B_{2}\right)$ defined over $|\Sigma|$.

Claim. There exists a unimodular fan $\Delta$ with $|\Delta|=|\Sigma|$ such that every $\ell$-function in $S \cup$ Hats $_{\Sigma}$ is linear over each cone of $\Delta$.

To prove this, let $\mathcal{L}_{1}$ be the collection of all linear pieces of all $\ell$-functions in $S \cup$ Hats $_{\Sigma}$, regarded by linear extension 11 as linear $\ell$-functions from $\mathbb{R}^{n}$ into itself. Further, let $\mathcal{L}_{2}$ be a collection of rational homogeneous hyperplanes in $\mathbb{R}^{n}$ defining the rational homogeneous polyhedral set $|\Sigma|$, again regarded as linear $\ell$-functions from $\mathbb{R}^{n}$ into itself. Set $\mathcal{L}=\mathcal{L}_{1} \cup \mathcal{L}_{2}$, say $|\mathcal{L}|=m$; display the elements of $\mathcal{L}$ as $\mathcal{L}=\left\{l_{1}, \ldots, l_{m}\right\}$. We construct a complete unimodular fan $\Xi$ in $\mathbb{R}^{n}$ as follows. For every permutation $\sigma$ of $\{1, \ldots, m\}$, set

$$
E_{\sigma}=\left\{\mathbf{x} \in \mathbb{R}^{n} \mid l_{\sigma(1)}(\mathbf{x}) \geq l_{\sigma(2)}(\mathbf{x}) \geq \cdots \geq l_{\sigma(m)}(\mathbf{x})\right\}
$$

Then $E_{\sigma} \subseteq \mathbb{R}^{n}$ is a rational homogeneous polyhedral set; we do not exclude the trivial case $E_{\sigma}=\{0\}$, in which case there is nothing to prove. In all remaining cases, the dimension of $E_{\sigma}$ is $n$. It is an exercise to check that there exists a unique refinement-maximal (not necessarily unimodular, nor even simplicial) fan $\Xi$ in $\mathbb{R}^{n}$ whose $n$-dimensional cones are precisely the nontrivial $E_{\sigma}$, as $\sigma$ ranges over all permutations of $\{1, \ldots, m\}$. Furthermore, it is clear that $\bigcup_{\sigma} E_{\sigma}=\mathbb{R}^{n}$, whence $\Xi$ is complete. By e.g. [6, Theorem 8.5], every fan can be unimodularised; let $\Theta$ be a unimodular refinement of $\Xi$. Now $|\Sigma|$ is inscribed in $\Theta$, meaning that $|\Sigma|$ is a union of cones of $\Theta$, because $\mathcal{L}$ includes $\mathcal{L}_{2}$, a collection of hyperplanes defining $\Sigma$. Let $\Delta$ be the fan obtained from $\Xi$ by selecting all those cones $\delta \in \Theta$ that intersect $|\Sigma|$ nontrivially, i.e. $\delta \cap|\Sigma| \neq\{0\}$. Then clearly $|\Delta|=|\Sigma|, \Delta$ is unimodular, and every $\ell$-function in $S \cup$ Hats $_{\Sigma}$ is linear over each cone of $\Delta$; the latter condition holds because $\mathcal{L}$ includes $\mathcal{L}_{2}$, the collection of all linear pieces of all $\ell$-functions in $S \cup$ Hats $_{\Sigma}$. This settles our first claim.

\footnotetext{
${ }^{11}$ Such an extension is generally not unique, but the choice of the extension is immaterial.
} 
Claim. Hats $\Delta=\left\{h_{1}, \ldots, h_{t}\right\}$ spans $S \cup$ Hats $_{\Sigma}$ positively.

Indeed, let $f:|\Sigma| \rightarrow \mathbb{R}$ be an $\ell$-function that is linear over each cone of $\Delta$. For each 1-cone $\delta_{i} \in \Delta, i \in\{1, \ldots, t\}, f$ attains an integral value $z_{i} \in \mathbb{Z}$ at the primitive vector along $\delta_{i}$. The $\ell$-function $\tilde{f}=z_{1} h_{1}+\cdots+z_{t} h_{t}$ coincides with $f$ : it indeed does by construction over 1-cones, hence it does over any cone because both $f$ and $\tilde{f}$ are linear over each cone of $\Delta$. This settles our second claim.

We thus have $S, \operatorname{Hats}_{\Sigma} \subseteq\left[\left[\operatorname{Hats}_{\Delta}\right]\right]$. Set $C=\phi^{-1}\left(\operatorname{Hats}_{\Delta}\right)$. Since $\phi^{-1}$ is an $\ell$-isomorphism, $B_{1}, B_{2} \subseteq[[C]]$ holds, and the proof is complete.

If $\Sigma$ refines $\Delta$, there is generally no way of obtaining $\Sigma$ from $\Delta$ via a sequence of starring operations; thus, $\Sigma \preceq_{2} \Delta$ implies $\Sigma \leq \Delta$, but not conversely. The stronger $\Sigma \preceq_{2} \Delta$ condition that $\Sigma$ be a starring refinement of $\Delta$ also has an algebraic

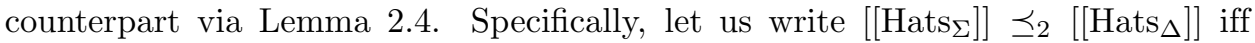
Hats $_{\Sigma}$ can be obtained from Hats $\Delta$ via a finite number of transformations of the type

$$
\left\{h_{1}, h_{2}\right\} \mapsto\left\{h_{1}-\left(h_{1} \wedge h_{2}\right), h_{2}-\left(h_{1} \wedge h_{2}\right), h_{1} \wedge h_{2}\right\},
$$

with the proviso that $h_{1} \wedge h_{2} \neq 0$. We can then prove the following purely algebraic version of the De Concini-Procesi Lemma.

Proposition 5.2 (The abstract De Concini-Procesi Lemma). Let $B_{1}, B_{2}$ be abstract Schauder bases of an $\ell$-group $G$. There exists an abstract Schauder basis $C$ of $G$ such that:

(1) $C \leq B_{2}$, in the sense that $B_{2} \subseteq[[C]]$.

(2) $C \preceq_{2} B_{1}$, in the sense that $C$ is obtainable from $B_{1}$ via a finite number of transformations of the type (4), where $h_{1} \wedge h_{2} \neq 0$.

Proof. Let $\phi: G \cong_{\ell} \mathcal{A}_{n} \uparrow|\Sigma|$ be an $\ell$-isomorphism carrying $B_{1}$ to Hats ${ }_{\Sigma}$, for some unimodular fan $\Sigma$ in $\mathbb{R}^{n}$, and let $S=\phi\left(B_{2}\right)$. By the same argument as the one in Proposition 5.1 there is a unimodular fan $\Delta$ with $|\Delta|=|\Sigma|$ such that $S \cup \operatorname{Hats}_{\Sigma} \subseteq$ $\left[\left[\right.\right.$ Hats $\left.\left._{\Delta}\right]\right]$. By the De Concini-Procesi Lemma, there is a unimodular fan $\Theta$ such that $\Theta \preceq_{2} \Sigma$, and $\Theta$ refines $\Delta$. It follows that Hats ${ }_{\Sigma}, S \subseteq\left[\left[\right.\right.$ Hats $\left.\left._{\Theta}\right]\right]$, because every $\ell$-function in $\operatorname{Hats}_{\Sigma} \cup S$ is linear over each cone of $\Delta$, hence over each cone of its refinement $\Theta$. Thus, if $C=\phi^{-1}\left(\operatorname{Hats}_{\Theta}\right)$, we have $B_{1}, B_{2} \subseteq[[C]]$. Since $\Theta \preceq_{2} \Sigma$, Lemma 2.4 shows Hats $\Theta$ is obtainable from Hats ${ }_{\Sigma}$ via a finite number of transformations of type (4), with $h_{1} \wedge h_{2} \neq 0$. Since these transformations are preserved by $\ell$-isomorphism, we obtain $C \preceq_{2} B_{1}$, which completes the proof.

The above result should be compared with Lemma 2.3, to which it reduces when $B_{1}$ and $B_{2}$ are concrete Schauder bases.

\section{Finitely Generated PROJECTIVE = PRESENTABlE By A LATtice WORD}

We refer to [7, Section 5.2], [13, Section 4] and [8 for background on finite presentations of $\ell$-groups. As an immediate consequence of Corollary 4.4 we have:

Proposition 6.1. Finitely presented $\ell$-groups $G \cong\left\langle\pi_{1}, \ldots, \pi_{m}: v=0\right\rangle$ are exactly the same as finitely generated projective $\ell$-groups. 
We are in a position to prove 12

Theorem 6.2. For any $\ell$-group $G$ the following are equivalent:

(1) $G$ is finitely generated projective.

(2) $G$ is finitely presented by $\left\langle\pi_{1}, \ldots, \pi_{n}: l=0\right\rangle$, where the $\ell$-function $l \in$ $\mathcal{A}_{n}$ is obtainable from $\pi_{1}, \ldots, \pi_{n}, 0$ by applying the lattice operations only.

Proof. $(2 \rightarrow 1)$ Follows immediately from Proposition 6.1.

$(1 \rightarrow 2)$ In light of Corollary 4.4 it is sufficient to prove the theorem for any $\ell$ group $G$ of the form $G=\mathcal{A}_{n}\left\lceil|\Theta|\right.$, where $\Theta$ is a (necessarily) unimodular fan in $\mathbb{R}^{n}$ whose 1-dimensional cones are generated by the standard basis vectors $\mathbf{e}_{1}, \ldots, \mathbf{e}_{n}$. In order to get the desired presentation of such a $G$ it is enough to exhibit an $\ell$-function $l \in \mathcal{A}_{n}$ having the following two properties:

- the vanishing locus $l^{-1}(0) \subseteq \mathbb{R}^{n}$ of $l$ coincides with $|\Theta|$, and

- $l$ is obtainable from $\pi_{1}, \ldots, \pi_{n}, 0$ by applying the lattice operations only.

To this purpose one first notes that $|\Theta|$ is contained in the positive orthant $\mathcal{O}$ of $\mathbb{R}^{n}$, and in addition, Hats $\Theta=\left\{\pi_{1} \uparrow|\Theta|, \ldots, \pi_{n} \uparrow|\Theta|\right\}$.

Let the $\ell$-function $w \in \mathcal{A}_{n}$ be defined by

$$
w=\bigvee_{i=1}^{n} \pi_{i} \wedge 0
$$

The vanishing locus of $w$ coincides with the intersection $\mathcal{O}$ of the vanishing loci of the $\ell$-functions $\pi_{i} \wedge 0$. If $|\Theta|=\mathcal{O}$ we are done. Otherwise, for every cone $\theta$ of $\Theta$, let $\mathbf{e}_{\theta, 1}, \ldots, \mathbf{e}_{\theta, r}$ be the primitive generating vectors of $\theta$, and let $\mathbf{e}_{\theta, r+1}, \ldots, \mathbf{e}_{\theta, n}$ be the remaining basis vectors of $\mathbb{R}^{n}$. Note that this latter set is nonempty. Let the $\ell$-function $l_{\theta}$ be defined by

$$
l_{\theta}=w \vee \pi_{\theta, r+1} \vee \cdots \vee \pi_{\theta, n} .
$$

Then the vanishing locus of $l_{\theta}$ coincides with $\theta$, as is immediately seen. Finally, let the $\ell$-function $l$ be defined by

$$
l=\bigwedge_{\theta \in \Theta} l_{\theta} .
$$

The vanishing locus of $l$ coincides with the union $|\Theta|$ of the cones of $\Theta$. This completes the proof.

Final remark. Suppose the finitely generated projective $\ell$-group $G$ is presented by $\left\langle\pi_{1}, \ldots, \pi_{m}: v=0\right\rangle$. Suppose the $\ell$-function $v$ is explicitly written as an $\ell$ group word $\omega$, i.e., as a string of symbols over the alphabet $\{0,+,-, \wedge, \vee\}$ of $\ell$ groups, plus symbols $X_{1}, \ldots, X_{m}$ for the variables 13 using the familiar composition rules. Then the proof of the theorem above yields an alternative presentation $G \cong\left\langle\pi_{1}, \ldots, \pi_{n}: l=0\right\rangle$, where the $\ell$-function $l$ can be explicitly written as a $\{0, \wedge, \vee\}$-word $\lambda$ in the variables $Y_{1}, \ldots, Y_{n}$, and the map $\omega \mapsto \lambda$ is effective 14

As a matter of fact, from $\omega\left(X_{1}, \ldots, X_{m}\right)$ one can effectively 15 construct a unimodular fan $\Delta$ whose support $|\Delta| \subseteq \mathbb{R}^{m}$ coincides with the vanishing locus of $v$.

\footnotetext{
${ }^{12}$ Compare with [10 page 47].

${ }^{13}$ Also, if needed, such orthographic symbols as parentheses and commas.

${ }^{14}$ Here, by abuse of notation, $\pi_{j}$ may denote a free generator both in $\mathcal{A}_{m}$ and in $\mathcal{A}_{n}$.

${ }^{15}$ See [13 Proposition 4.3] for details.
} 
Let $\mathbf{p}_{1}, \ldots, \mathbf{p}_{n}$ display the primitive generating vectors of the rays in $\Delta$. By sending each $\mathbf{p}_{i}$ to the standard basis vector $\mathbf{e}_{i}$ of $\mathbb{R}^{n}$ one effectively obtains from $\Delta$ a unimodular fan $\Theta$ in $\mathbb{R}^{n}$ : the cones in $\Delta$ precisely correspond to the cones in $\Theta$ via the map $\mathbf{p}_{i} \mapsto \mathbf{e}_{i}$. The desired lattice-word $\lambda$ is now effectively obtainable from $\Theta$ as in the proof of Theorem 6.2 The $\ell$-isomorphisms

$$
\mathcal{A}_{m} \uparrow|\Delta| \cong \mathcal{A}_{n} \uparrow|\Theta| \cong\left\langle\pi_{1}, \ldots, \pi_{m}: v=0\right\rangle \cong\left\langle\pi_{1}, \ldots, \pi_{n}: l=0\right\rangle
$$

follow from the trivial piecewise homogeneous linear homeomorphism existing between $|\Delta|$ and $|\Theta|$ in the light of Baker-Beynon duality (see [7, 5.2]).

\section{ACKNOWLEDGEMENTS}

The second author was partially supported by the Research Training Network COMBstru (Combinatorial Structure of Intractable Problems) of the European Community, contract no. HPRN-CT-2002-00278.

The first and second authors feel deeply indebted to Stefano Aguzzoli for several conversations on the subject of this paper. In particular, an early statement of Corollary 4.4 with a self-contained proof, emerged during those conversations.

The third author is indebted to B. J. Totaro for background on singular homology groups.

\section{REFERENCES}

[1] K. A. Baker. Free vector lattices, Canad. J. Math. 20:58-66, 1968. MR0224524 (37:123)

[2] W. M. Beynon. Duality theorems for finitely generated vector lattices, Proc. London Math. Soc. (3) 31:114-128, 1975. MR0376480(51:12655)

[3] W. M. Beynon. Applications of duality in the theory of finitely generated lattice-ordered abelian groups, Canad. J. Math. 29(2):243-254, 1977. MR0437420 (55:10350)

[4] A. Bigard, K. Keimel and S. Wolfenstein. Groupes et Anneaux Réticulés, volume 608 of Lecture Notes in Mathematics. Springer-Verlag, Berlin, 1971. MR.0552653 (58:27688)

[5] C. De Concini and C. Procesi. Complete symmetric varieties. II. Intersection theory. In Kyoto and Nagoya, editors, Algebraic groups and related topics, volume 6 of Advanced Studies in Pure Mathematics, pages 481-513. North-Holland, Amsterdam, 1985. MR0803344 (87a:14038)

[6] G. EWALD. Combinatorial convexity and algebraic geometry, volume 168 of Graduate Texts in Mathematics. Springer-Verlag, New York, 1996. MR,1418400 (97i:52012)

[7] A. M. W. Glass. Partially ordered groups, volume 7 of Series in Algebra. World Scientific, Singapore, 1999. MR 1791008 (2001g:06002)

[8] A. M. W. Glass, V. Marra. Embedding finitely generated abelian lattice-ordered groups: Highman's theorem and a realisation of $\pi$, J. London Math. Soc. (2) 68:545-562, 2003. MR2009436 (2004h:06017)

[9] C. MAnaRA. Relating the theory of non-Boolean partitions to the design of interpretable control systems. Ph.D. thesis, Università degli Studi di Milano, 2003.

[10] V. Marra. Non-Boolean partitions. A mathematical investigation through lattice-ordered Abelian groups and MV algebras. Ph.D. thesis, Università degli Studi di Milano, 2002.

[11] W. S. Massey. Singular Homology Theory, volume 70 of Graduate Texts in Mathematics. Springer-Verlag, New York, 1980. MR0569059 (81g:55002)

[12] D. Mundici. Farey stellar subdivisions, ultrasimplicial groups and $K_{0}$ of $\mathrm{AF} C^{*}$-algebras, Advances in Mathematics 68:23-39, 1988. MR0931170 (89d:46072)

[13] D. Mundici. Simple Bratteli diagrams with a Gödel-incomplete $C^{*}$-equivalence problem, Trans. Amer. Math. Soc. 356(5):1937-1955, 2004. MR2031047(2004k:46103)

[14] D. Mundici. A characterization of free $n$-generated MV-algebras. Archive Mathematical Logic 45:239-247, 2006.

[15] T. OdA. Convex Bodies and Algebraic Geometry. Springer-Verlag, Berlin, 1988. MR0922894 (88m:14038) 
[16] G. Panti. A geometric proof of the completeness of the Eukasiewicz calculus, J. Symbolic Logic 60(2):563-578, 1995. MR1335137 (96h:03058)

[17] Z. SEMAdENI. Schauder bases in Banach spaces of continuous functions, volume 918 of Lecture Notes in Mathematics. Springer-Verlag, Berlin, 1982. MR0653986 (83g:46023)

Via Pellicioli 10, 24127 Bergamo, Italy

E-mail address: corrado.manara@gmail.com

Dipartimento di Informatica e Comunicazione, Università degli Studi di Milano, via Comelico 39/41, I-20135 Milano, Italy

E-mail address: marra@dico.unimi.it

Dipartimento di Matematica "Ulisse Dini", Università degli Studi di Firenze, viale Morgagni 67/A, I-50134 Firenze, Italy

E-mail address: mundici@math.unifi.it 